\title{
Long non-coding RNA PRNCR1 has an oncogenic role in breast cancer
}

\author{
QIAN GUO ${ }^{1 *}$, SHUANG LV $^{2 *}$, BINGPING WANG ${ }^{3}$, YINBING LI $^{1}$, NIER CHA ${ }^{1}$, \\ RUIGANG ZHAO $^{1}$, WENHUA BAO ${ }^{1}$ and BAOQING JIA ${ }^{1}$ \\ ${ }^{1}$ Breast Neoplasms Surgical Department; ${ }^{2}$ Department of Oncology; ${ }^{3}$ Oncology Institute, \\ Inner Mongolia People's Hospital, Huhehot, Inner Mongolia 010010, P.R. China
}

Received December 31, 2018; Accepted July 16, 2019

DOI: $10.3892 /$ etm.2019.8152

\begin{abstract}
Long non-coding RNAs (lncRNAs) have important roles in the development and progression of various types of human cancer. However, the expression and function of the lncRNA prostate cancer-associated non-coding RNA 1 (PRNCR1) in breast cancer remains unclear. Reverse transcription-quantitative PCR was performed to measure the levels of mRNA expression. Cell counting kit- 8 , flow cytometry, wound healing and Transwell assays were also performed to study cell proliferation, cell cycle, migration and invasion, respectively. The results of the present study revealed that PRNCR1 expression levels were higher in breast cancer tissues compared with adjacent normal tissues in a patient study. It was also determined that high expression of PRNCR1 was significantly associated with advanced clinical stage, positive metastasis and poor prognosis for patients with breast cancer. In vitro experiments determined that PRNCR1 was significantly upregulated in the breast cancer cell lines BT-549, MCF-7, SK-BR-3 and MDA-MB-231 compared with the normal human breast cell line, MCF-10A. Silencing of PRNCR1 significantly inhibited the proliferation, colony formation, cell cycle progression, migration and invasion of SK-BR-3 and BT-549 cells, while cell apoptosis was induced. In addition, knockdown of PRNCR1 suppressed epithelial-mesenchymal transition in SK-BR-3 and BT-549 cells. In summary, the present results demonstrated that lncRNA PRNCR1 was significantly upregulated in breast cancer and was associated with cancer progression and poor patient prognosis. In vitro experiments determined that knockdown of PRNCR1 inhibited the malignant phenotypes of breast cancer cells. Taken together, the results indicated that PRNCR1
\end{abstract}

Correspondence to: Dr Baoqing Jia, Breast Neoplasms Surgical Department, Inner Mongolia People's Hospital, 20 Zhaowuda Road, Huhehot, Inner Mongolia 010010, P.R China

E-mail: 13604715646@qq.com

${ }^{*}$ Contributed equally

Key words: long non-coding RNA, prostate cancer-associated non-coding RNA 1, breast cancer may be used as a potential therapeutic target for patients with breast cancer.

\section{Introduction}

Breast cancer is the most common malignant tumor of females and is the leading cause of cancer-associated death in women, worldwide (1). Despite great improvements in surgical resection combined with advances in chemotherapy and radiotherapy, the overall survival time for patients with advanced stage breast cancer remains very poor, primarily due to the rapid proliferation and metastasis of cancer cells (1-3). Understanding the molecular mechanisms underlying breast cancer growth and metastasis is important, as it may contribute to the discovery of novel molecular targets for breast cancer treatment $(4,5)$.

Long non-coding RNAs (lncRNAs) are a class of non-coding RNAs > 200 nucleotides in length that have been demonstrated to be involved in various physiological and pathological processes by regulating cell proliferation, apoptosis, cell cycle progression, migration and invasion $(3,6,7)$. Recently, increasing numbers of IncRNAs have been identified to be deregulated in various malignant tumors, including breast cancer $(8,9)$. Furthermore, IncRNAs have been reported to serve important roles in cancer by affecting the malignant phenotypes of breast cancer cells (3,9-11). For instance, the lncRNA nuclear enriched abundant transcript 1 (NEAT1) is significantly upregulated in breast cancer, whilst knockdown of NEAT1 significantly inhibits the growth of breast cancer cells (10). The 1ncRNA small nucleolar RNA host gene 20 promotes the proliferation, invasion and migration of breast cancer cells by inhibiting the expression of microRNA (miR)-495 (9). The downregulation of lncRNA growth arrest-specific 5 induces trastuzumab resistance in breast cancer (11).

Prostate cancer-associated non-coding RNA 1 (PRNCR1) is localized to chromosome $8 \mathrm{q} 24$ and was originally reported to be associated with prostate and colorectal cancer susceptibility $(12,13)$. PRNCR1 directly binds to androgen receptors (ARs) to enhance ligand-dependent and -independent AR gene expression as well as the proliferation of prostate cancer cells (14). Yang et al (15) reported that PRNCR1 is upregulated in colorectal cancer and promotes cancer cell proliferation 
and cell cycle progression. Cheng et al (16) identified that PRNCR1 upregulated hes related family bHLH transcription factor with YRPW motif 2 to promote non-small-cell lung carcinoma (NSCLC) progression by competitively binding miR-448 (16). In addition to being associated with cancer, PRNCR1 also affects osteogenic differentiation and contributes to osteolysis following hip replacement (17). Additionally, PRNCR1 promotes the progression of eclampsia by regulating the mitogen activated kinase-like signal pathway (18).

However, the detailed role of PRNCR1 in breast cancer remains unknown. Therefore, the present study aimed to examine the clinical significance of PRNCR1 expression in breast cancer and to explore the role of PRNCR1 in breast cancer cell proliferation, apoptosis, migration and invasion. The findings may provide a potential therapeutic target for patients with breast cancer.

\section{Materials and methods}

Clinical tissue samples. A total of 52 paired breast cancer tissues and adjacent normal tissues, which were cut $\geq 3 \mathrm{~cm}$ from cancer tissues, were obtained from 52 female patients (mean age, 54.4 \pm 13.5 years; age range, 33-76 years) with primary breast cancer at the Department of Breast Neoplasm Surgery, Inner Mongolia People's Hospital between June 2012 and September 2013 (Table I). The present study was approved by the Ethics Committee of Inner Mongolia People's Hospital and written consent was obtained from all participants. The inclusion criteria were that these patients were primary patients with breast cancer. Patients with preoperative chemotherapy and/or radiotherapy were excluded from the current study. Luminal cancers were defined based on PAM50 (19). These tissues were immediately snap-frozen in liquid nitrogen following surgery and stored at $-80^{\circ} \mathrm{C}$ until required. The patients were followed up once every 2-3 months post-surgery for 5 years.

Cell culture. A normal human breast cell line, MCF-10A, and 4 human breast cancer cell lines, BT-549, MCF-7, SK-BR-3 and MDA-MB-231, were obtained from Cell Bank of Type Culture Collection of Chinese Academy of Sciences. All cell lines were cultured in DMEM (Thermo Fisher Scientific, Inc.) with $10 \%$ FBS (Thermo Fisher Scientific, Inc.) at $37^{\circ} \mathrm{C}$ with $5 \% \mathrm{CO}_{2}$.

Cell transfection. Cell transfection was performed using Lipofectamine ${ }^{\circledR} 2000$ (Thermo Fisher Scientific, Inc.) in accordance with the manufacturer's protocol. SK-BR-3 and BT-549 cells were transfected with $100 \mathrm{nM}$ scrambled (NC) small interfering (si)RNA (cat. no. 12935200, Thermo Fisher Scientific, Inc.), PRNCR1 siRNA1 (cat. no. n541275, Thermo Fisher Scientific, Inc.) and PRNCR1 siRNA2 (cat. no. n541276, Thermo Fisher Scientific, Inc.). The following experiments were performed after cell transfection for $48 \mathrm{~h}$.

Reverse transcription-quantitative PCR (RT-qPCR) assay. Total RNA was extracted from cells or tissues using TRIzol ${ }^{\circledR}$ reagent (Thermo Fisher Scientific, Inc.). cDNA was synthesized from $1 \mu \mathrm{g}$ RNA using a PrimeScript RT Master Mix kit (Takara Bio, Inc.) in accordance with the manufacturer's protocols. qPCR was subsequently performed to determine mRNA expression using SYBR Green qPCR Master Mix (Thermo Fisher Scientific, Inc.) on an ABI 7300 Plus Real-Time PCR System (Thermo Fisher Scientific, Inc.). The thermocycling conditions were as follows: $95^{\circ} \mathrm{C}$ for $30 \mathrm{sec}$, followed by 40 cycles of $95^{\circ} \mathrm{C}$ for $15 \mathrm{sec}$ and $60^{\circ} \mathrm{C}$ for $30 \mathrm{sec}$. The relative mRNA expression was quantified by the $2^{-\Delta \Delta C a}$ method and normalized to GAPDH (20). The primers for GAPDH were forward, 5'-GGAGCGAGATCCCTCCAAAAT-3' and reverse, 5'-GGCTGTTGTCATACTTCTCATGG-3'. The primers for PRNCR1 were forward, 5'-CCAGATTCCAAGGGCTGA TA-3' and reverse, 5'-GATGTTTGGAGGCATCTGGT-3'.

Western blot analysis. Transfected SK-BR-3 and BT-549 cells were lysed using radioimmunoprecipitation assay buffer (Beyotime Institute of Biotechnology). Proteins (50 $\mu \mathrm{g}$ per lane) were separated via SDS-PAGE on a $10 \%$ gel then transferred onto polyvinylidene difluoride membranes (Thermo Fisher Scientific, Inc.). The membranes were blocked with 5\% dry milk in Tris-buffered saline (TBS) with $0.2 \%$ of Tween-20 at $4^{\circ} \mathrm{C}$ overnight. The membranes were subsequently incubated with primary antibodies against E-cadherin (1:500; ab15148; Abcam), N-cadherin (1:500; ab18203; Abcam), Vimentin (1:500; ab8978; Abcam), and GAPDH (1:250; ab9485; Abcam) at room temperature for $3 \mathrm{~h}$. Membranes were then incubated with horseradish peroxidase-conjugated secondary antibody $(1: 5,000 ;$ ab6721; Abcam) at room temperature for $1 \mathrm{~h}$. The protein signals were detected using an Enhanced Chemiluminescence Western Blotting Substrate kit (Thermo Fisher Scientific, Inc.) and quantified using ImageJ software v1.46 (National Institutes of Health). GAPDH was used as an internal control.

CCK-8 assay. Transfected SK-BR-3 and BT-549 cells were resuspended in DMEM with 10\% FBS and seeded into 96-well plates $\left(10,000\right.$ cells per well). Following incubation at $37^{\circ} \mathrm{C}$ for $0,24,48$ or 72 h, $10 \mu 1$ CCK-8 reagent (Thermo Fisher Scientific, Inc.) was added to cells. Then cells were incubated at $37^{\circ} \mathrm{C}$ for another $2 \mathrm{~h}$. The optical density absorbance at $450 \mathrm{~nm}$ was measured using a Varioskan LUX Multimode Microplate Reader (Thermo Fisher Scientific, Inc.) according to the manufacturer's protocols.

Colony formation assay. Transfected SK-BR-3 and BT-549 cells were resuspended in DMEM with 10\% FBS and seeded into 6-well plates ( 150 cells/well). Cells were then cultured at $37^{\circ} \mathrm{C}$ for 14 days. Colonies were washed with PBS, fixed with $10 \%$ formalin at room temperature for $10 \mathrm{~min}$ and stained with $0.1 \%(\mathrm{w} / \mathrm{v})$ crystal violet (Amresco LLC) at room temperature for $10 \mathrm{~min}$. The number of cell colonies (containing $>50$ cells) were counted using a light microscope (magnification, $\mathrm{x} 40$ ).

Cell apoptosis assay. Cell apoptosis was analyzed using an Annexin V-fluorescein isothiocyanate (FITC) Apoptosis kit (BD Biosciences) in accordance with the manufacturer's protocol. Transfected SK-BR-3 and BT-549 cells were stained with Annexin V-FITC and propidium iodide (PI) solutions at $4^{\circ} \mathrm{C}$ for $30 \mathrm{~min}$. Then, the cell apoptosis rate was detected by a FACSCalibur flow cytometer (Becton, Dickinson and Company) and analyzed using BD Accuri C6 software (version 1.0; Becton, Dickinson and Company) . 
Table I. Association between PRNCR1 expression and clinicopathological characteristics in patients with breast cancer.

\begin{tabular}{|c|c|c|c|c|}
\hline Variables & Number $(\mathrm{n}=52)$ & Low expression $(n=29)$ & High expression $(n=23)$ & P-value \\
\hline Age (years) & & & & 0.680 \\
\hline$\leq 50$ & 22 & 13 & 9 & \\
\hline$>50$ & 30 & 16 & 14 & \\
\hline Subtype & & & & 0.591 \\
\hline Lunimal A type & 28 & 18 & 10 & \\
\hline Lunimal B type & 5 & 2 & 3 & \\
\hline HER2 positive & 8 & 4 & 4 & \\
\hline TNBC & 11 & 5 & 6 & \\
\hline Differentiation & & & & 0.077 \\
\hline Well and moderately & 36 & 23 & 13 & \\
\hline Poor & 16 & 6 & 10 & \\
\hline Lymph node metastasis & & & & $0.017^{\mathrm{a}}$ \\
\hline Present & 38 & 25 & 13 & \\
\hline Absent & 14 & 4 & 10 & \\
\hline Distant metastasis & & & & $0.004^{\mathrm{a}}$ \\
\hline Present & 6 & 0 & 6 & \\
\hline Absent & 46 & 29 & 17 & \\
\hline TNM stage & & & & $0.008^{\mathrm{a}}$ \\
\hline I-II & 35 & 24 & 11 & \\
\hline III-IV & 17 & 5 & 12 & \\
\hline
\end{tabular}

${ }^{\mathrm{a}} \mathrm{P}<0.05$. HER2, human epidermal growth factor receptor 2; TNBC, triple-negative breast cancer; TNM, TNM Classification of Malignant Tumors.

Cell cycle analysis. Transfected SK-BR-3 and BT-549 cells were cultured in DMEM with $10 \% \mathrm{FBS}$ at $37^{\circ} \mathrm{C}$ for $24 \mathrm{~h}$. Cells were fixed in $70 \%$ ethanol at $4^{\circ} \mathrm{C}$ overnight and incubated in $1 \mathrm{ml}$ PBS with $0.1 \%$ TritonX-100, $60 \mu \mathrm{g} / \mathrm{ml} \mathrm{PI}$, $0.1 \mathrm{mg} / \mathrm{ml}$ DNase free RNase and $0.1 \%$ trisodium citrate at $4^{\circ} \mathrm{C}$ for $30 \mathrm{~min}$. Cell cycle distribution was measured using a FACSCalibur flow cytometer and analyzed using BD Accuri C6 software 1.0 (Becton, Dickinson and Company).

Wound healing assay. After transfection for $48 \mathrm{~h}$, a wound healing assay was performed to assess cell migration. A wound was introduced to transfected SK-BR-3 and BT-549 cells with a sterile $100 \mu \mathrm{l}$ micropipette tip. Cells were then washed twice with PBS to remove cellular debris and cultured at $37^{\circ} \mathrm{C}$ in serum-free DMEM for $24 \mathrm{~h}$. Cells were photographed using an inverted microscope (IX71; Olympus Corporation; magnification, $\mathrm{x} 40$ ) at 0 and $24 \mathrm{~h}$, and the wound width was analyzed using ImageJ software 1.46 (National Institutes of Health).

Transwell assay. A transwell assay was performed to assess cell invasion. Transfected SK-BR-3 and BT-549 cells $(10,000$ cells/well) were resuspended in $200 \mu 1$ serum-free DMEM, which were then added into the upper chambers (pore size; $8 \mu \mathrm{m}$ ) precoated with Matrigel (EMD Millipore; Merck $\mathrm{KGaA})$. The lower chambers were filled with $500 \mu 1 \mathrm{DMEM}$ with $10 \%$ FBS. Cells were then incubated at $37^{\circ} \mathrm{C}$ for $48 \mathrm{~h}$. A cotton swab was used to carefully remove non-invading cells from the top chamber. Cells on the lower surface were fixed in
$75 \%$ ethanol at room temperature for $30 \mathrm{~min}$ and stained with $0.1 \%$ crystal violet at room temperature for $5 \mathrm{~min}$. Invasive cells were photographed and counted under a phase-contrast inverted microscope (magnification, x200).

Statistical analysis. All experiments were performed in triplicate. Data are presented as the mean \pm standard deviation and analyzed using GraphPad Prism 5 (GraphPad Software, Inc.). Student's t-tests were used to analyze differences between two groups and one-way analysis of variance followed by a Tukey's post-hoc test was used for the comparisons of multiple groups. A $\chi^{2}$ test was used for analyzing the association between PRNCR1 expression and clinical characteristics of breast cancer patients. Survival analysis was conducted using Kaplan-Meier survival curves and a log-rank test. $\mathrm{P}<0.05$ was considered to indicate a statistically significant difference.

\section{Results}

Upregulation of PRNCRI in breast cancer predicts poor prognosis. A qPCR assay was performed to determine the expression of PRNCR1 in breast cancer cell lines and the normal human breast cell line, MCF-10A. The expression of PRNCR1 was significantly higher in breast cancer cell lines compared with MCF-10A cells (Fig. 1A). To confirm these findings in vivo, PRNCR1 expression in 52 paired breast cancer tissues and adjacent normal tissues were investigated. It was demonstrated that PRNCR1 was significantly upregulated 
A

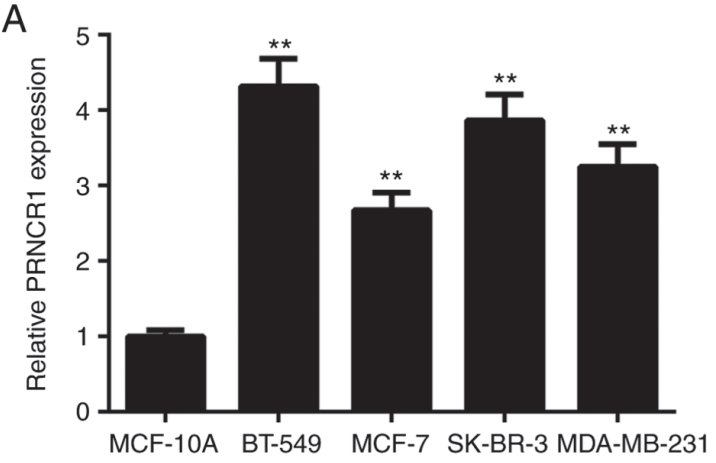

B

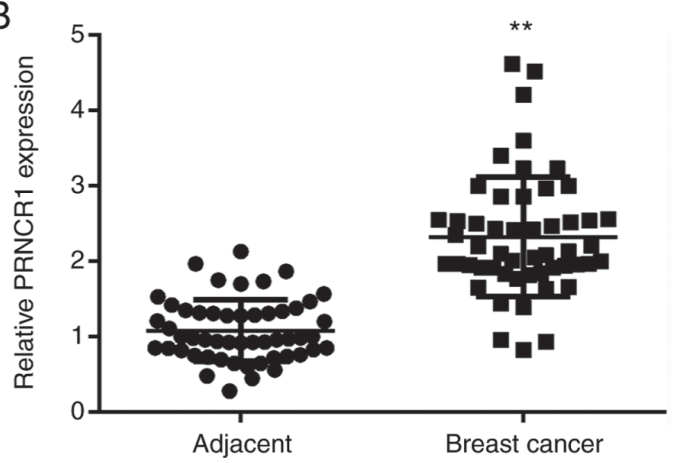

- ᄂ. Low PRNCR1 expression

+ High PRNCR1 expression

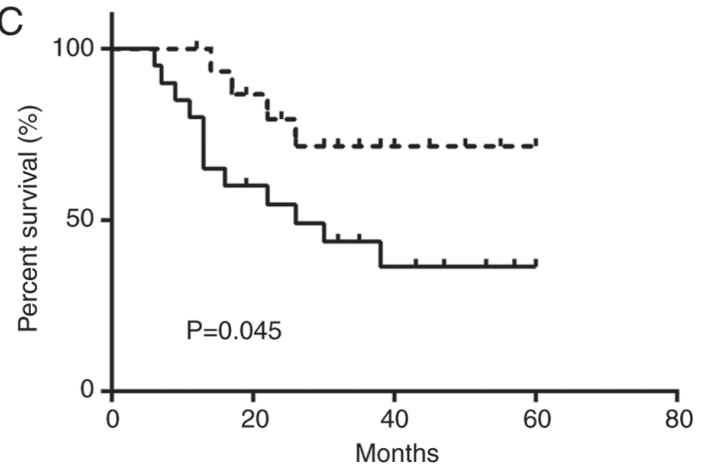

Figure 1. Overexpression of PRNCR1 predicted poor prognosis in breast cancer. (A) RT-qPCR demonstrated that PRNCR1 was significantly upregulated in breast cancer cell lines compared with the normal human cancer cell line, MCF-10A. ${ }^{* *} \mathrm{P}<0.01$ vs. MCF-10A. (B) RT-qPCR demonstrated that PRNCR1 was significantly upregulated in breast cancer tissue compared with adjacent normal tissue. ${ }^{* *} \mathrm{P}<0.01$ vs. adjacent. (C) Breast cancer patients with a high PRNCR1 expression exhibited worse prognoses than those with a low expression of PRNCR1 over a 5-year period. PRNCR1, prostate cancer-associated non-coding RNA 1; RT-qPCR, reverse transcription-quantitative PCR.

in breast cancer tissues compared with adjacent normal tissues (Fig. 1B). The 52 patients with breast cancer were divided into low and high PRNCR1 expression groups based on the median expression level (median value, 2.38) of PRNCR1 as a cutoff. Further investigation demonstrated that high PRNCR1 expression was significantly associated with positive metastasis and advanced TNM Classification of Malignant Tumors (TNM) stage (Table I), indicating that the upregulation of PRNCR1 may serve a role in breast cancer progression. Survival analysis indicated that the patients with high PRNCR1 expression exhibited shorter survival times compared with those with low PRNCR1 expression (Fig. 1C). The results indicated that a high upregulation of PRNCR1 predicts a poor prognosis in patients with breast cancer.

PRNCR1 silencing inhibits breast cancer cell proliferation and colony formation. The role of PRNCR1 in breast cancer in vitro was assessed in the current study. SK-BR-3 and BT-549 cells were transfected with NC siRNA or two different PRNCR1 siRNAs, respectively. As presented in Fig. 2A, RT-qPCR data indicated that PRNCR1 levels were significantly decreased following transfection with PRNCR1 siRNA1 or siRNA2 compared with the NC siRNA group. As PRNCR1 siRNA1 demonstrated the highest inhibitory effect on the expression of PRNCR1 in SK-BR-3 and BT-549 cells, it was selected for subsequent experimentation. A CCK-8 assay was then performed to assess the effects of PRNCR1 downregulation on breast cancer cell proliferation. The proliferation of SK-BR-3 and BT-549 cells was significantly reduced in the
PRNCR1 siRNA group compared with the NC siRNA group (Fig. 2B and C). A colony formation assay was subsequently performed, which determined that the colony formation capacities of SK-BR-3 and BT-549 cells were significantly inhibited following the silencing of PRNCR1 expression (Fig. 2D).

PRNCRI knockdown represses cell cycle progression and promotes SK-BR-3 and BT-549 cell apoptosis. The present study hypothesized that the role of PRNCR1 in breast cancer cell proliferation may occur by affecting cell cycle progression and/or cell apoptosis. Thus, flow cytometry was performed to determine the effects of PRNCR1 downregulation on cell cycle progression and apoptosis in SK-BR-3 and BT-549 cells. The percentage of SK-BR-3 and BT-549 cells in the G1 stage was significantly increased, while the percentage of cells in the G2/M stage was significantly decreased in the PRNCR1 siRNA group compared with the NC siRNA group (Fig. 3A and B). These results indicated that the silencing of PRNCR1 caused a cycle arrest at the G1 stage in SK-BR-3 and BT-549 cells. Furthermore, the apoptotic rate of SK-BR-3 and BT-549 cells was significantly higher in the PRNCR1 siRNA group compared with the NC siRNA group (Fig. 3C and D). Therefore, the knockdown of PRNCR1 was demonstrated to inhibit cell cycle progression and promote the apoptosis of breast cancer cells.

PRNCR1 knockdown inhibits migration, invasion and epithelial-mesenchymal transition (EMT) of breast cancer cells. The function of PRNCR1 in in vitro breast cancer 

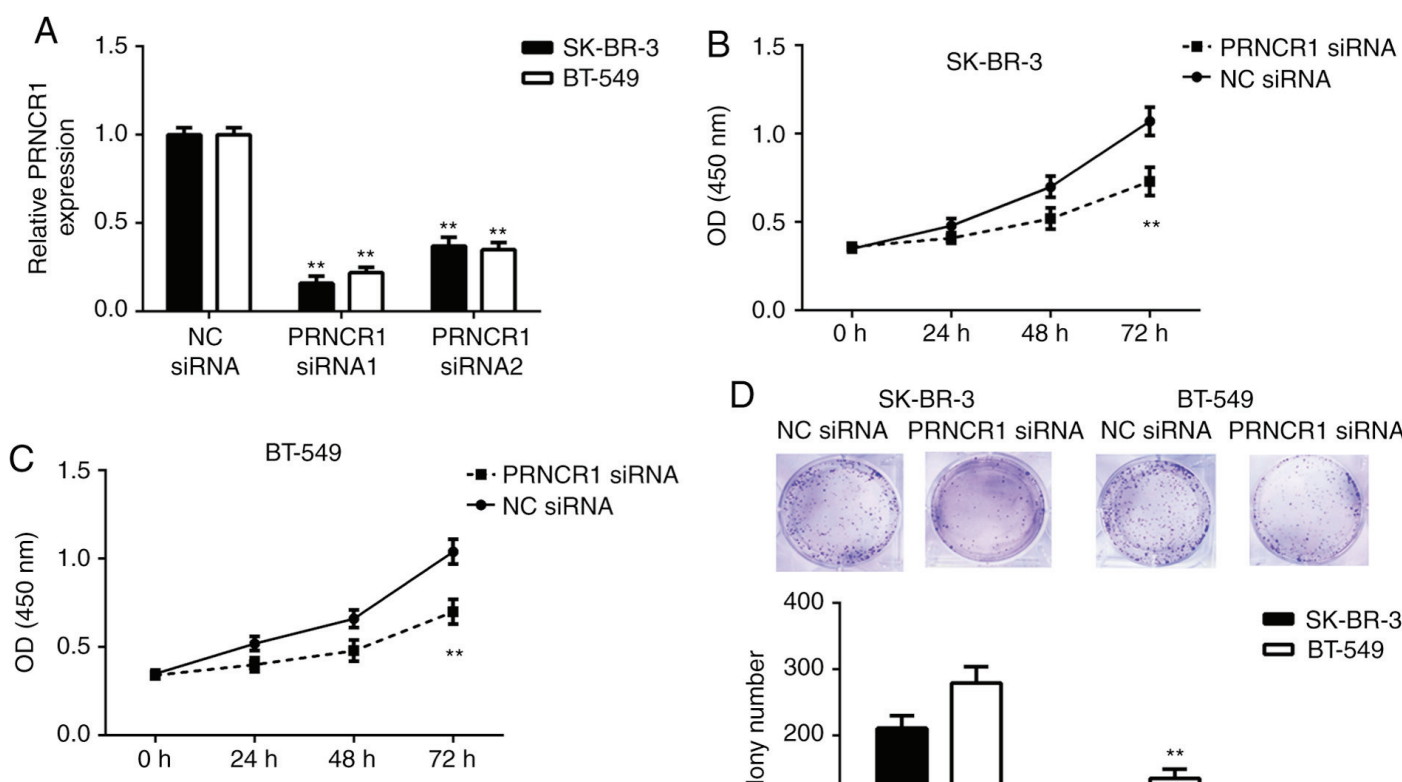

D SK-BR-3
NC SiRNA PRNC 1 SiRNA NC SiRNA PRNCR1 siRNA

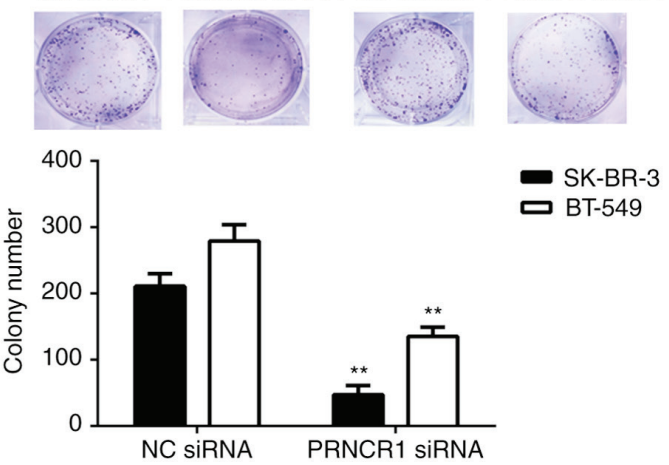

Figure 2. Knockdown of PRNCR1 inhibited breast cancer cell proliferation and colony formation. (A) SK-BR-3 and BT-549 cells were transfected with NC siRNA, PRNCR1 siRNA1 or PRNCR1 siRNA2 with transfection efficiency successfully demonstrated. (B) A cell counting kit-8 assay demonstrated that cell proliferation in SK-BR-3 and (C) BT-549 cells was decreased following PRNCR1 knockdown. (D) A colony formation assay demonstrated that colony number decreased following PRNCR1 knockdown. ${ }^{* *} \mathrm{P}<0.01$ vs. NC siRNA. PRNCR1, prostate cancer-associated non-coding RNA 1; NC, negative control; siRNA, small interfering RNA; OD, optical density.
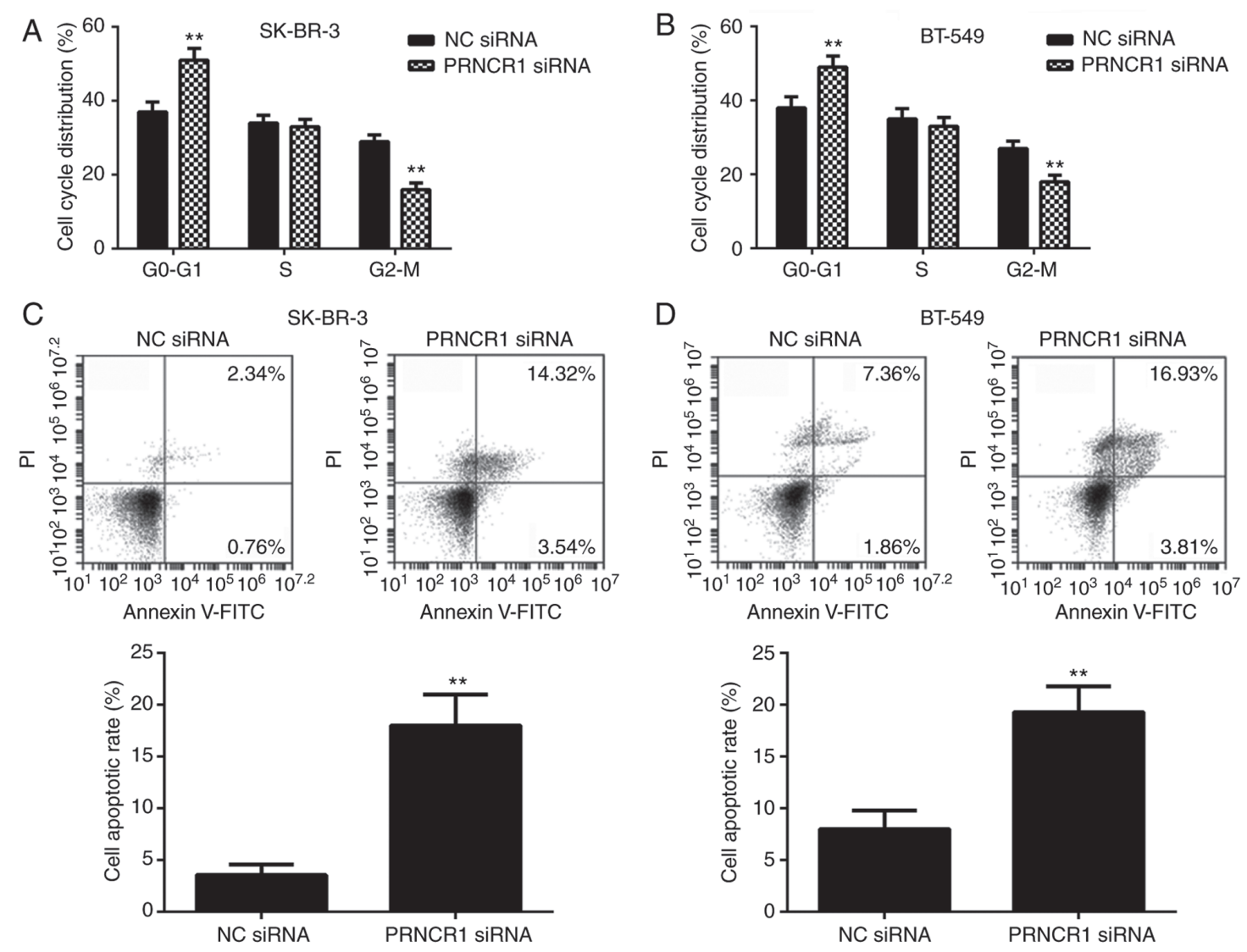

Figure 3. Inhibition of PRNCR1 induced cell cycle arrest and cell apoptosis in breast cancer. (A) SK-BR-3 and (B) BT-549 cells were transfected with NC siRNA or PRNCR1 siRNA, after which cell cycle distribution was determined using flow cytometry. Cell apoptosis was determined using flow cytometry in (C) SK-BR-3 and (D) BT-549 cells. ${ }^{* *}$ P $<0.01$ vs. NC siRNA. PRNCR1, prostate cancer-associated non-coding RNA 1; NC, negative control; siRNA, small interfering RNA; PI, propidium iodide; FITC, fluorescein isothiocyanate. 
A
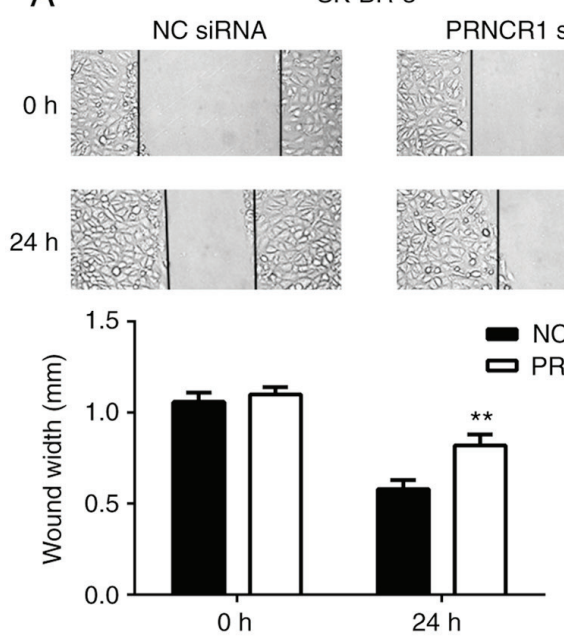

C
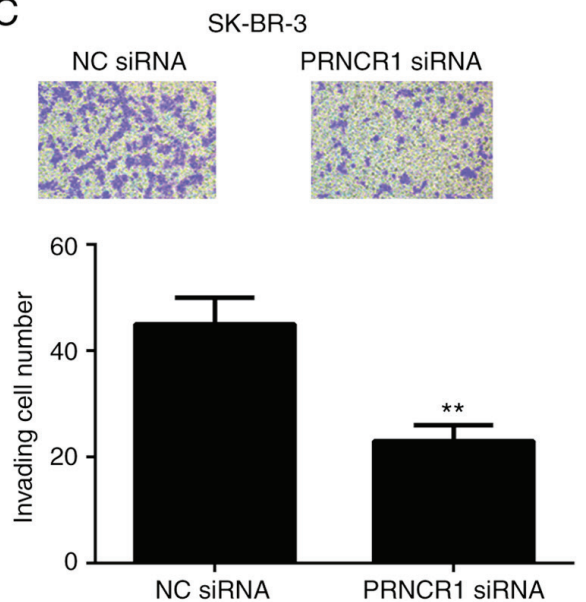

B PRNCR1 SiRNA
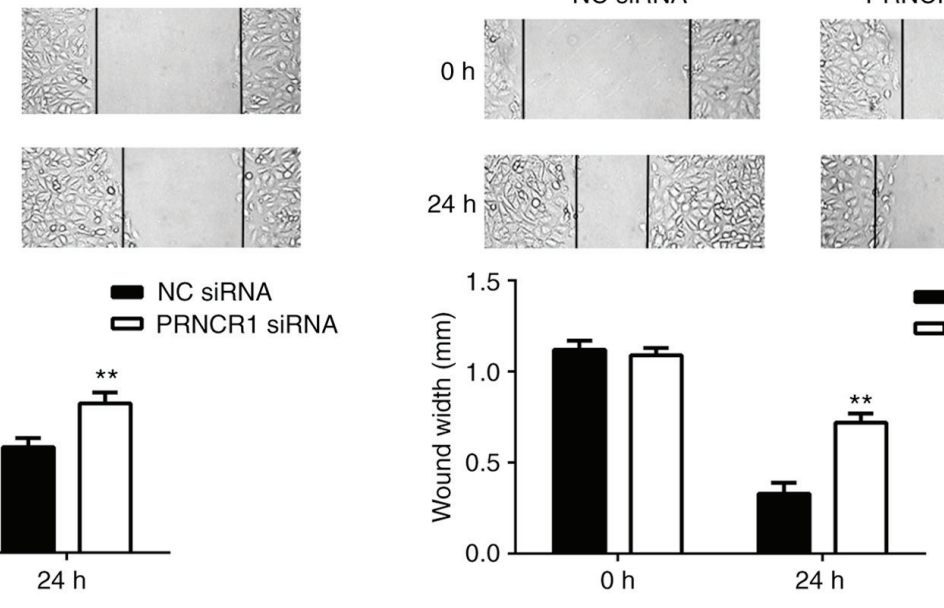

D
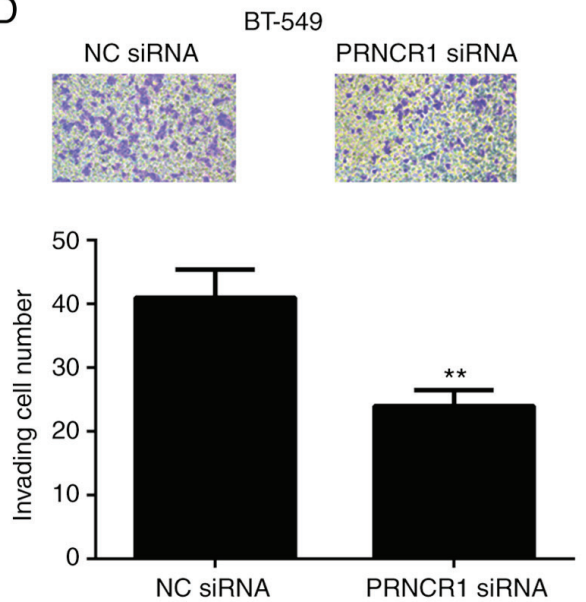

Figure 4. Inhibition of PRNCR1 decreased breast cancer cell migration and invasion. (A) SK-BR-3 and (B) BT-549 cells were transfected with NC siRNA or PRNCR1 siRNA then wound healing assay was performed to determine cell migration. (C) Transwell assay were used to examine cell invasion in SK-BR-3 and (D) BT-549 cells. ${ }^{* *} \mathrm{P}<0.01$ vs. NC siRNA. PRNCR1, prostate cancer-associated non-coding RNA 1; NC, negative control; siRNA, small interfering RNA.

metastasis was assessed. Wound healing and Transwell assays were performed to examine the effects of PRNCR1 downregulation on the migration and invasion of breast cancer cells. The results indicated that the migration of SK-BR-3 and BT-549 cells was significantly decreased following PRNCR1 silencing compared with the control group (Fig. 4A and B). In agreement, the invasive capacity of cells was also significantly reduced in the PRNCR1 siRNA group compared to the NC siRNA group (Fig. 4C and D). These results indicated that PRNCR1 may have a promoting role in breast cancer metastasis.

EMT is essential for cancer cell migration and invasion (21). Therefore, the effects of PRNCR1 on the EMT of breast cancer cells were determined. The protein expressions of E-cadherin (an epithelial marker), N-cadherin (a mesenchymal marker) and vimentin (a mesenchymal marker) were examined in SK-BR-3 and BT-549 cells. Silencing of PRNCR1 expression resulted in a significant increase in E-cadherin expression with significant reductions in $\mathrm{N}$-cadherin and vimentin compared with the NC siRNA group (Fig. 5). Taken together, these results indicated that the inhibition of PRNCR1 repressed the migration and invasion of breast cancer cells by downregulating EMT.

\section{Discussion}

To the best of our knowledge, the expression and function of lncRNA PRNCR1 in breast cancer has not previously been investigated. In the present study, it was determined that PRNCR1 was significantly upregulated in breast cancer, with high expression of PRNCR1 significantly associated with breast cancer progression and poor prognosis. For in vitro experiments, the knockdown of PRNCR1 inhibited breast cancer cell proliferation, colony formation, cell cycle progression, migration, invasion, EMT and promoted breast cancer cell apoptosis.

Recently, accumulating evidence has revealed that IncRNAs serve key roles in breast cancer progression, with certain IncRNAs exhibiting potential as biomarkers and therapeutic targets for this disease (22). For instance, the IncRNA, DSCAM Antisense RNA 1, regulates the G1/S cell cycle transition and is an independent prognostic factor for poor survival in luminal breast cancer patients treated with endocrine therapy (22). Knockdown of the lncRNA, HOX transcript antisense RNA, sensitized breast cancer cells to ionizing radiation by activating miR-218 (23). The lncRNA, long intergenic non-protein coding RNA 1116, has 

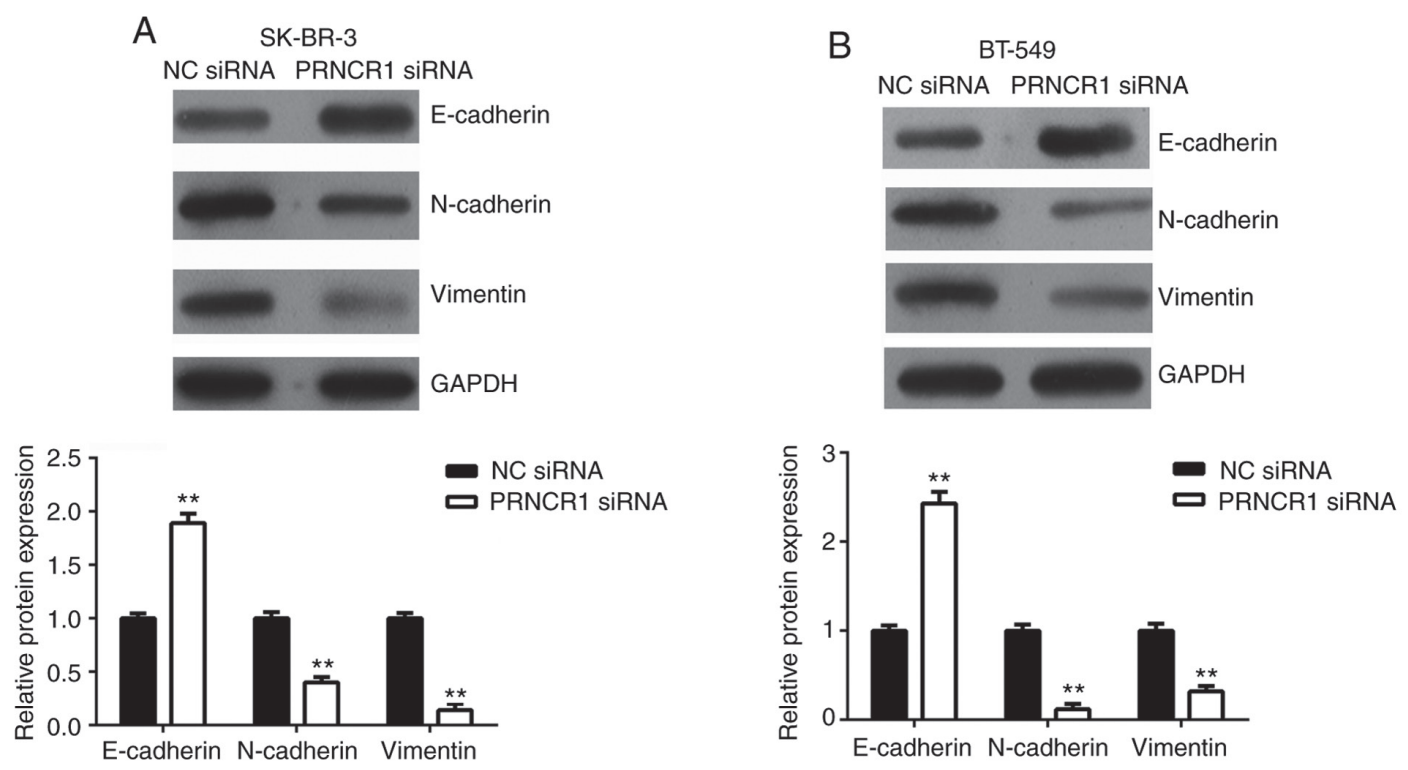

Figure 5. Effects of PRNCR1 downregulation on epithelial-mesenchymal transition in breast cancer cells. (A) SK-BR-3 and (B) BT-549 cells were transfected with NC siRNA or PRNCR1 siRNA, after which the protein expression of E-cadherin, N-cadherin and vimentin was determined via western blotting. ${ }^{* *} \mathrm{P}<0.01$ vs. NC siRNA. PRNCR1, prostate cancer-associated non-coding RNA 1; NC, negative control; siRNA, small interfering RNA.

an oncogenic role in breast cancer by binding to enhancer of zeste 2 polycomb repressive complex 2 subunit to target p57 and is involved in the mitogen activated protein kinase and NF- $\kappa \mathrm{B}$ signaling pathways (24). However, no previous study has focused on the expression of PRNCR1 in breast cancer. The present study observed that PRNCR1 was significantly upregulated in breast cancer cell lines and clinical tissues compared with normal breast MCF-10A cells and adjacent normal tissues, respectively. In addition, it was identified that the increased expression of PRNCR1 was significantly associated with positive lymph node metastasis and advanced TNM stage in breast cancer patients, indicating that upregulation of PRNCR1 promoted breast cancer progression. Furthermore, patients with high PRNCR1 expression exhibited shorter survival times compared with those with low PRNCR1 expressions, indicating that the expression of PRNCR1 may be used as a promising predicator of prognosis in breast cancer.

The role of PRNCR1 in breast cancer progression in vitro was investigated via a loss-of-function assay in SK-BR-3 and BT-549 cells. The results demonstrated that PRNCR1 silencing led to a significant reduction in breast cancer cell proliferation and colony formation. These results indicated that PRNCR1 may have a promoting role in breast cancer growth. As the cell cycle is essential for cell proliferation, the function of PRNCR1 in cell cycle progression in breast cancer cells was investigated. The results determined that silencing of PRNCR1 expression led to significant cell cycle arrest in the G1 stage. This indicated that the suppressive effect of PRNCR1 downregulation on breast cancer cell proliferation may be due to cell cycle arrest in the G1 stage. Similarly, Yang et al (15) demonstrated that PRNCR1 upregulation promotes colorectal cancer cell proliferation and cell cycle progression. In addition, it was observed that the cell apoptosis rate was significantly increased following silencing of PRNCR1 in breast cancer cells, which may also be involved in the downregulation of cell proliferation induced by PRNCR1 inhibition.
The function of PRNCR1 in breast cancer metastasis was assessed in the current study, as tumor cell migration and invasion are crucial for tumor metastasis (21). The results demonstrated that knockdown of PRNCR1 inhibited breast cancer cell migration and invasion. These results indicated that PRNCR1 may serve a promoting role in breast cancer metastasis. Thus, future studies should be performed to further confirm the function of PRNCR1 in breast cancer metastasis in vivo via animal experiments. EMT is characterized by the loss of an epithelial phenotype and acquisition of mesenchymal properties. It has been widely accepted that EMT is essential for cell migration and invasion, and thus serves a crucial role in cancer metastasis (25). Previous studies have identified that lncRNAs are involved during the EMT process in different types of human cancer. For instance, the lncRNA, X-inactive specific transcript, promotes TGF- $\beta$-induced EMT by regulating the miR-367/141-zinc finger E-box binding homeobox 2 axis in NSCLC (21). The lncRNA, colorectal neoplasia differentially expressed, enhances EMT in intrahepatic cholangiocarcinoma (26). However, to the best of our knowledge, no previous study has reported the effects of PRNCR1 on EMT. In the present study, it was identified that the inhibition of PRNCR1 significantly increased E-cadherin expression while decreasing $\mathrm{N}$-cadherin and vimentin expressions, indicating that EMT was suppressed. These results suggested that PRNCR1 may have promoting effects on EMT in breast cancer cells and that the inhibitory effect of PRNCR1 knockdown in breast cancer cell migration and invasion may be due to the inhibition of EMT. The limitations of the present study are the lack of a mechanistic assessment. Therefore, future studies should involve RNA sequencing followed by the knockdown of PRNCR1 in breast cancer cells to analyze the pathways involved in PRNCR1 regulation, as well as EMT. In addition, the targets of PRNCR1 in breast cancer should also be investigated. 
To the best of our knowledge, the present study was the first to report that the lncRNA PRNCR1 was significantly upregulated in breast cancer. The study also determined that PRNCR1 was strongly associated with cancer progression and poor patient prognosis. In vitro experiments identified that the knockdown of its expression effectively inhibited malignant phenotypes of breast cancer cells. Taken together, these results suggested that PRNCR1 could be used as a potential therapeutic target for breast cancer.

\section{Acknowledgements}

Not applicable.

\section{Funding}

No funding was received.

\section{Availability of data and materials}

The datasets used and/or analyzed during the current study are available from the corresponding author on reasonable request.

\section{Authors' contributions}

BJ designed the study. RZ and WB collected clinical tissues and performed the statistical analyses. QG, SL, BW, YL and NC performed the experiments. QG and SL and wrote the manuscript. All authors read and approved the final manuscript.

\section{Ethics approval and consent to participate}

The present study was approved by the Ethics Committee of Inner Mongolia People's Hospital. All patients provided written informed consents.

\section{Patient consent for publication}

Not applicable.

\section{Competing interests}

The authors declare that they have no competing interests.

\section{References}

1. Siegel RL, Miller KD and Jemal A: Cancer statistics, 2017. CA Cancer J Clin 67: 7-30, 2017.

2. Vishal M, Swetha R, Thejaswini G, Arumugam B and Selvamurugan N: Role of Runx2 in breast cancer-mediated bone metastasis. Int J Biol Macromol 99: 608-614, 2017.

3. Xu S, Kong D, Chen Q, Ping Y and Pang D: Oncogenic long noncoding RNA landscape in breast cancer. Mol Cancer 16: 129, 2017.

4. Luo T, Yan Y, He Q, Ma X and Wang W: miR-328-5p inhibits MDA-MB-231 breast cancer cell proliferation by targeting RAGE. Oncol Rep 39: 2906-2914, 2018.

5. Zhou Y, Meng X, Chen S, Li W, Li D, Singer R and Gu W: IMP1 regulates UCA1-mediated cell invasion through facilitating UCA1 decay and decreasing the sponge effect of UCA1 for miR-122-5p. Breast Cancer Res 20: 32, 2018.

6. Peng Z, Liu C and Wu M: New insights into long noncoding RNAs and their roles in glioma. Mol Cancer 17: 61, 2018.
7. Taylor DH, Chu ET, Spektor R and Soloway PD: Long non-coding RNA regulation of reproduction and development. Mol Reprod Dev 82: 932-956, 2015.

8. Wang O, Yang F, Liu Y, Lv L, Ma R, Chen C, Wang J, Tan Q, Cheng Y, Xia E, et al: C-MYC-induced upregulation of IncRNA SNHG12 regulates cell proliferation, apoptosis and migration in triple-negative breast cancer. Am J Transl Res 9: 533-545, 2017.

9. Guan YX, Zhang ZM, Chen XZ, Zhang Q, Liu SZ and Zhang YL: Lnc RNA SNHG20 participated in proliferation, invasion and migration of breast cancer cells via miR-495. J Cell Biochem 119: 7971-7981, 2018.

10. Jiang X, Zhou Y, Sun AJ and Xue JL: NEAT1 contributes to breast cancer progression through modulating miR-448 and ZEB1. J Cell Physiol 233: 8558-8566, 2018.

11. Li W, Zhai L, Wang H, Liu C, Zhang J, Chen W and Wei Q: Downregulation of LncRNA GAS5 causes trastuzumab resistance in breast cancer. Oncotarget 7: 27778-27786, 2016.

12. Chung S, NakagawaH,Uemura M,Piao L, Ashikawa K, Hosono N, Takata R, Akamatsu S, Kawaguchi T, Morizono T, et al: Association of a novel long non-coding RNA in 8q24 with prostate cancer susceptibility. Cancer Sci 102: 245-252, 2011.

13. Li L, Sun R, Liang Y, Pan X, Li Z, Bai P, Zeng X, Zhang D, Zhang $\mathrm{L}$ and Gao L: Association between polymorphisms in long non-coding RNA PRNCR1 in 8q24 and risk of colorectal cancer. J Exp Clin Cancer Res 32: 104, 2013.

14. Pestell RG and Yu Z: Long and noncoding RNAs (lnc-RNAs) determine androgen receptor dependent gene expression in prostate cancer growth in vivo. Asian J Androl 16: 268-269, 2014.

15. Yang L, Qiu M, Xu Y, Wang J, Zheng Y, Li M, Xu L and Yin R: Upregulation of long non-coding RNA PRNCR1 in colorectal cancer promotes cell proliferation and cell cycle progression. Oncol Rep 35: 318-324, 2016.

16. Cheng D, Bao C, Zhang X, Lin X, Huang H and Zhao L: LncRNA PRNCR1 interacts with HEY2 to abolish miR-448-mediated growth inhibition in non-small cell lung cancer. Biomed Pharmacother 107: 1540-1547, 2018.

17. Gong ZM, Tang ZY and Sun XL: LncRNA PRNCR1 regulates CXCR4 expression to affect osteogenic differentiation and contribute to osteolysis after hip replacement. Gene 673: 251-261, 2018.

18. Jiao S, Wang SY and Huang Y: LncRNA PRNCR1 promoted the progression of eclampsia by regulating the MAPK signal pathway. Eur Rev Med Pharmacol Sci 22: 3635-3642, 2018.

19. Liu MC, Pitcher BN, Mardis ER, Davies SR, Friedman PN, Snider JE, Vickery TL, Reed JP, DeSchryver K, Singh B, et al: PAM50 gene signatures and breast cancer prognosis with adjuvant anthracycline- and taxane-based chemotherapy: Correlative analysis of C9741 (Alliance). NPJ Breast Cancer 2: e15023, 2016.

20. Livak KJ and Schmittgen TD: Analysis of relative gene expression data using real-time quantitative PCR and the 2(-Delta Delta C(T)) method. Methods 25: 402-408, 2001.

21. Li C, Wan L, Liu Z, Xu G, Wang S, Su Z, Zhang Y, Zhang C, Liu X, Lei Z and Zhang HT: Long non-coding RNA XIST promotes TGF- $\beta$-induced epithelial-mesenchymal transition by regulating miR-367/141-ZEB2 axis in non-small-cell lung cancer. Cancer Lett 418: 185-195, 2018.

22. Sun W, Li AQ, Zhou P, Jiang YZ, Jin X, Liu YR, Guo YJ, Yang WT, Shao ZM and Xu XE: DSCAM-AS1 regulates the G1/S cell cycle transition and is an independent prognostic factor of poor survival in luminal breast cancer patients treated with endocrine therapy. Cancer Med 7: 6137-6146, 2018.

23. Hu X, Ding D, Zhang J and Cui J: Knockdown lncRNA HOTAIR sensitize breast cancer cells to ionizing radiation through activating miR-218. Biosci Rep 39: BSR20181038, 2019.

24. Qiao E, Chen D, Li Q, Feng W, Yu X, Zhang X, Xia L, Jin J and Yang H: Long noncoding RNA TALNEC2 plays an oncogenic role in breast cancer by binding to EZH2 to target p57(KIP2) and involving in p-p38 MAPK and NF- $\kappa$ B pathways. J Cell Biochem 120: 3978-3988, 2019.

25. Smith BN and Bhowmick NA: Role of EMT in metastasis and therapy resistance. J Clin Med 5: E17, 2016.

26. Xia XL, Xue D, Xiang TH, Xu HY, Song DK, Cheng PG and Wang JQ: Overexpression of long non-coding RNA CRNDE facilitates epithelial-mesenchymal transition and correlates with poor prognosis in intrahepatic cholangiocarcinoma. Oncol Lett 15: 4105-4112, 2018.

This work is licensed under a Creative Commons Attribution-NonCommercial-NoDerivatives 4.0 International (CC BY-NC-ND 4.0) License. 